\title{
Some features of gastric lymph glands in female and male cats of the British breed
}

\author{
M. S. Korableva*, L.V. Tkachenko, O.E. Maltseva, N.B. Kochetygova, and S.V. Burtseva \\ FSBEI of HE “Altai State Agrarian University”, Russia, Barnaul,
}

\begin{abstract}
The lymphatic system is directly involved in the gastric diseases development in animals. Pathologies of the gastrointestinal tract are in one of the first places for female cats. The purpose of the research: to study the features of topography and morphometry of gastric lymph glands in female cats 3-6 years of age. Objects of research were stomachs and gastric lymph glands from British breed female cats: 10 - aged 3-5 years, 10 - aged 3-6 years. The gastric lymph gland (right and/or left) was recorded in $80 \%$ of the animals studied. The shape was oblong, oval, and round. Dimensions: right $10,3 \pm 7,7 \mathrm{~mm}$, left $8,4 \pm 6,8 \mathrm{~mm}$. Features: absent in $20 \%$ of the studied male cats, can be horseshoe-shaped, spindle-shaped, can be double and lobular. The right lymph gland is slightly larger than the left. The duodenal lymph gland was recorded in $20 \%$ of animals. The shape was round. Dimensions: $12 \pm 12 \mathrm{~mm}$. Features: found only in female cats, having round shape and large size. The splenic lymph gland was noted in $30 \%$ of animals. Shape: oblong and round. Dimensions: $14,7 \pm 14,7$ $\mathrm{mm}$. Features: registered only in male cats, has elongated shape, the largest in the group of gastric lymph glands.
\end{abstract}

\section{Introduction}

The role of the lymphatic system in the vital activity of the body is very large. The lymphatic system is a powerful biological barrier; it shapes blood formed elements; participates in metabolism, transport of hormones, enzymes, vitamins; tissue nutrition [14].

There is evidence on the direct involvement of the immune system in the gastric diseases' development [1]. Pathologies of the gastrointestinal tract in female cats occupy one of the first places [2-5]. In the works of such authors as Akaevsky A.I. et al. (2009), Slesarenko N.A. (2003), the material was presented on the example of dogs and farm animals. The description of topography, name, age, breed, gender features were not found in the available literature, while Zelenevsky N.V. (2004) describes the general features of gastric lymph glands. Also, there was no exact international veterinary classification given, where.

Information of Konenkov V.I. (2012) indicates that there are lymphatic connections between the lymph glands of the stomach and other adjacent organs (spleen, pancreas, duodenum). Even though these are human data, we assume that this approach is

\footnotetext{
*Corresponding author: kirarrrrrr@yandex.ru
} 
anatomically sound and is acceptable to describe the lymph glands' characteristics of a female cat's stomach. Based on knowledge about the topography peculiarities and morphometric data of gastric lymph glands, a whole arsenal of diagnostic and therapeutic techniques is used in practice [6-9].

In this regard, we have set the following research goal: to study the features of topography and morphometry of gastric lymph glands in female cats of the British breed at the age of 3-6 years.

Objects of research were stomachs and gastric lymph glands from 10 female and 10 male cats aged 3-6 years, British breed.

\section{Methods of research}

1. Animals' registration in the "Registration Journal" with the date, animal type, age, gender, breed, and notes [10].

2. Postmortem examination by the Shor method with a description according to the generally accepted scheme [11].

3. Description of lymph glands topography: considering the features of the anatomictopographic location of the lymph gland, blood vessels, adjacent organs indicating the number of lymph glands, forms. [12].

4. Photographing of lymph glands in different projections [10].

5. Morphometric studies with statistical data processing, analysis of obtained results [13].

\section{Results and Discussion}

The group of gastric lymph glands include: right and left gastric lymph gland, splenic lymph gland, duodenal lymph gland [14].

Gastric lymph gland.

In this age group's female cats, $90 \%$ of cases the right and in $100 \%$ - the left gastric lymph gland was noted (Figure 2).

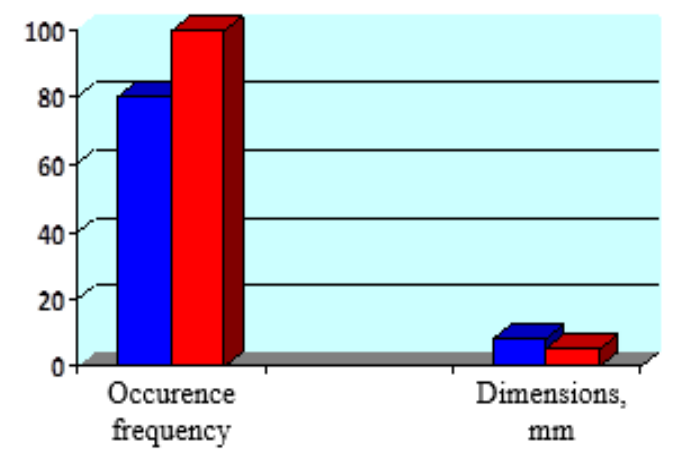

Gastric lymph gland in male cats

$\square$ Gastric lymph gland in

female cats

Fig. 1. Frequency of gastric lymph glands' occurrence in female and male cats.

Shape: mostly oblong, oval, and round (Figure 2).

Dimensions: right gastric lymph gland $-8.4 \pm 7 \mathrm{~mm}$, left $-7 \pm 5.5 \mathrm{~mm}$.

Features: a combination of different forms of the right and left lymph gland, large size.

There were gastric lymph glands observed in $80 \%$ of the studied male cats.

Shape: oblong, oval-round, and spindle-shaped. 
Dimensions: right gastric lymph gland - $8.5 \pm 4.9 \mathrm{~mm}$, left - $4.6 \pm 4.6 \mathrm{~mm}$

Features: mostly oval and round shape. The lymph gland size varied significantly, while the right lymph gland was larger than the left.
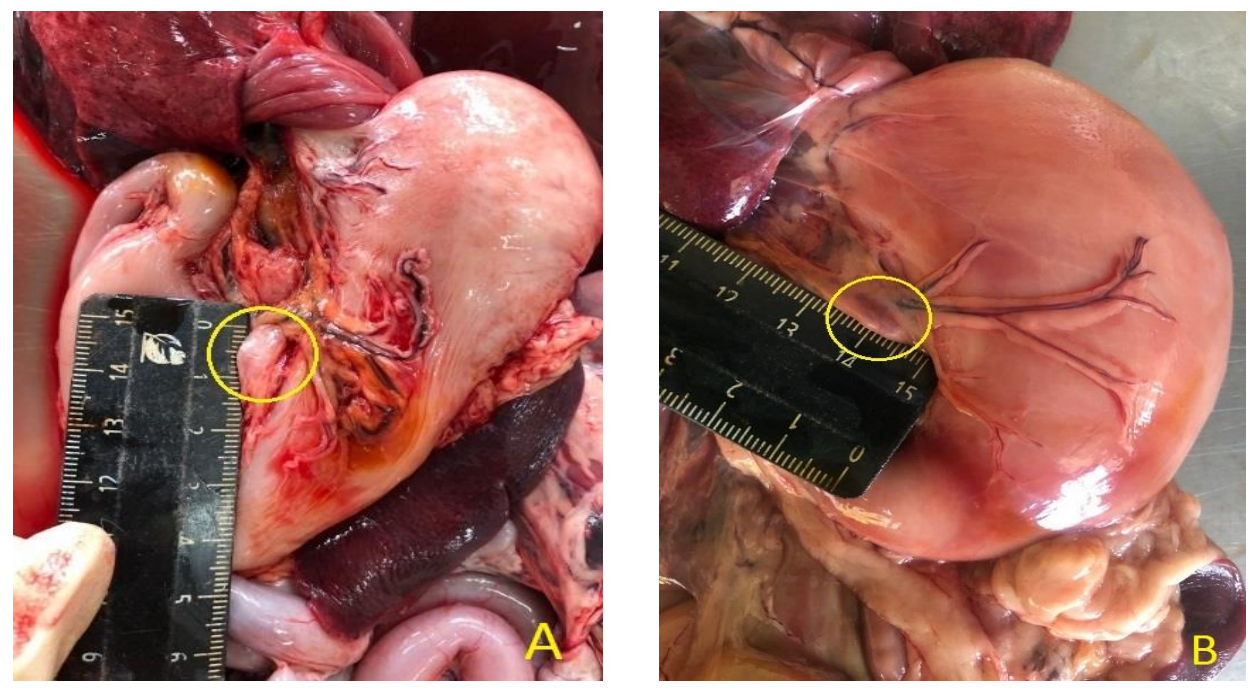

Fig. 2. Group of gastric lymph glands.

A. Right lymph gland. Female cat, 3 years old. British breed.
B. Left lymphatic gland. Male cat, 6 years old. British breed.

We believe that the typical structure and features of gastric lymph glands described above are explained by their anatomic-topographic location, namely the proximity of the liver, small intestine loops, and pancreas.

Duodenal lymph gland (Figure 3)

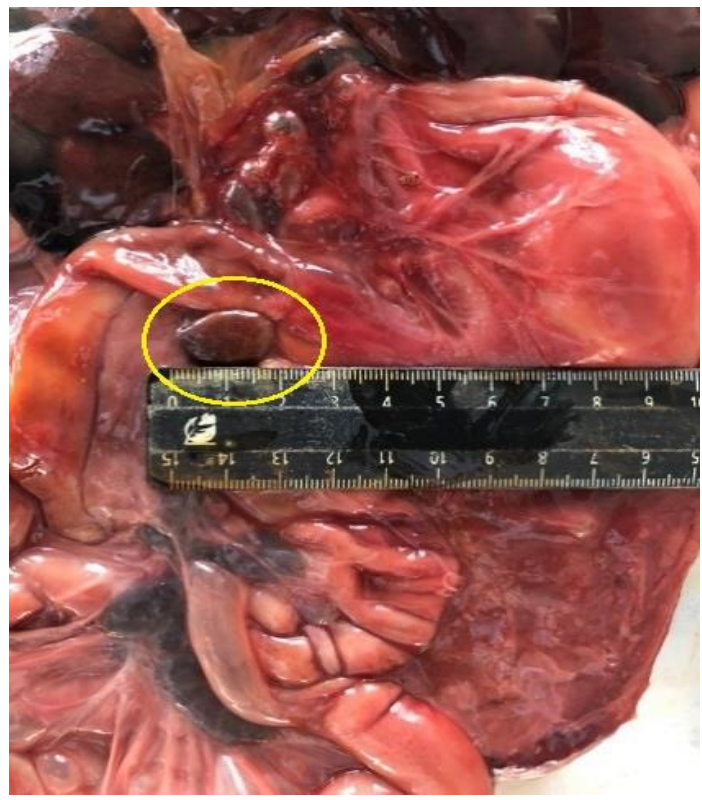

Fig. 3. Duodenal lymph gland. Female cat, 5 years old. British breed. 
In female cats, this lymph gland was recorded in $40 \%$ of animals (Figure 4 ).

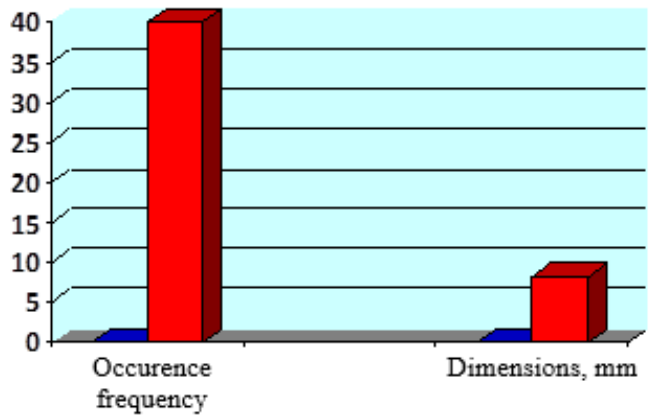

Duodenal lymph gland in male cats

$\square$ Duodenal lymph gland in

female cats

Fig. 4. Frequency of duodenal lymph glands occurrence in male and female cats.

The shape was round.

Dimensions: $8,25 \pm 8,25 \mathrm{~mm}$.

Features of the duodenal lymph gland: were found exclusively in female cats, only round shape, large size.

In male cats, this lymph gland is absent.

The anatomical proximity presence of stomach, liver, intestine and outflow pathways to the stomach explain the features of this lymph glands' group.

Splenic lymph gland (Figure 5)

In female cats, it was noted in $20 \%$ of animals (Figure 6).

The shape is oblong or oblong with a grainy surface.

Dimensions: $9 \pm 4,5 \mathrm{~mm}$.

Features of the splenic lymph gland: occurs in a small number of animals, only an oblong shape.

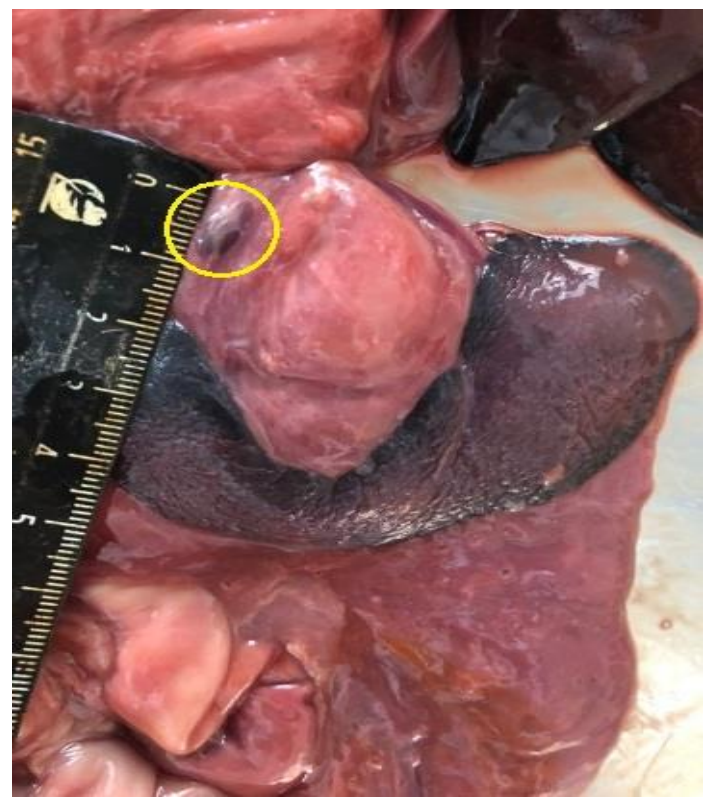

Fig. 5. Splenic lymph gland. Male cat, 3 years old. British breed.

In male cats: registered in $30 \%$ of animals. 
Shape: oval and oblong

Dimensions: $14,7 \pm 12,6 \mathrm{~mm}$

Features of the splenic lymph gland: this group of lymph glands is rarely recorded, mainly oblong in shape, have the maximum size in the group of gastric lymph glands.

The anatomical proximity presence of intestinal loops and outflow pathways to the duodenum explain the features of this lymph glands' group.

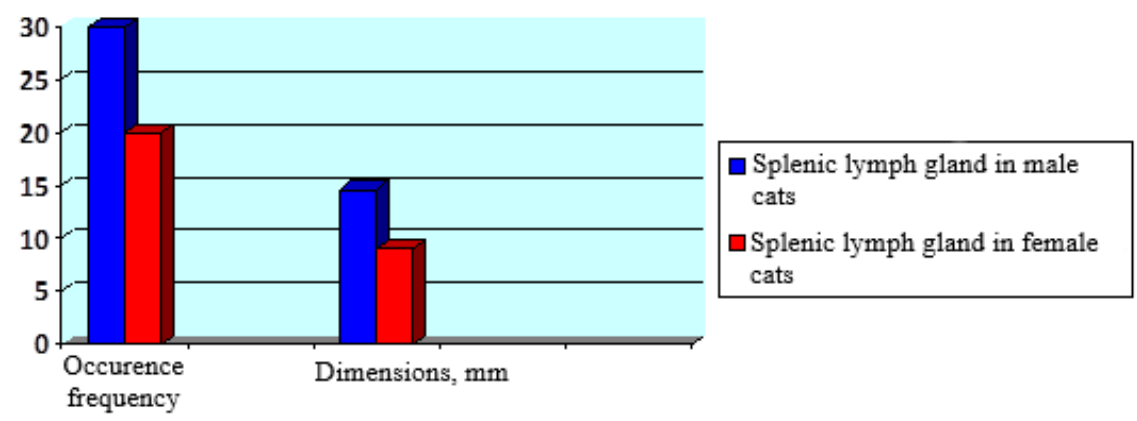

Fig. 6. Frequency of splenic lymph glands occurrence in male and female cats

\section{Conclusions}

1. The group of gastric lymph glands include: right and left gastric, splenic, duodenal lymph glands. The presence, size and shape of the lymph glands depend on gender and age.

2. Gastric lymph gland: registered in almost $100 \%$ of cases in female cats, oblong, oval, and round shape, large size. In male cats - recorded in $20 \%$ of cases, oblong, oval-rounded, and spindle-shaped, the right lymph gland is much larger than the left lymph gland.

3. Duodenal lymph gland: in female cats was recorded in $40 \%$ of cases, round shape, large size. In male cats, this lymph gland is absent.

4. Splenic lymph gland: noted in $20 \%$ of female cats, oblong shape or oblong with a grainy surface, large size. In male cats - registered in 30\%, shape: oval and oblong with the maximum size in the group of gastric lymph glands.

5. Presence, size and shape of the lymph glands depend on sex attribute, age, gender, and anatomic-topographic location.

\section{References}

1. I.I. Pavlova, "Mechanisms of body's physiological systems regulation in the adaptation process to the conditions of the environment", dedicated to the 85th anniversary of the foundation of the Institute of Physiology named after I.P.Pavlov, RAS, 148 (2010)

2. K.S. Ksenofontova, In the world of scientific discoveries, Proceedings of the III International Student Scientific Conference, 243-246 (2019)

3. A.V. Sapozhnikov, E.M. Maryin, P.M. Lyashenko, Bulletin of the Ulyanovsk State Agricultural Academy, 4 (32), 143-146 (2015)

4. V.M. Smirnov, I.L. Miasnikov, E.A. Berseneva, D.S. Sveshnikov, Russian journal of physiology, 1095-1102 (1999)

5. E.Yu. Kiselyova, A.A. Darbinyan, Scientific aspect, 12 (2), 1514-1517 (2019) 
6. D.S. Sveshnikov, V.M. Smirnov, I.L. Myasnikov, Bulletin of experimental biology and medicine, 5-8 (2011)

7. M.V. Inzhuvatova, K.O. Novikova, T.E. Vlasova, A.V. Sapozhnikov, A.V. Kireev, Students' Scientific Forum, VIII International Students' On-line Scientific Conference, Electronic Edition (2016)

8. O.A. Balabanova, K.A. Sidorova, N.A. Tatarnikova, Perm Agrarian Bulletin, 4 (24), $110-115$ (2018)

9. A.I. Zeibel, S.V. Pozyabin, Veterinary medicine, zootechnics and biotechnology, 1, 6$11(2016)$

10. M.S. Kirnos, L.V. Tkachenko, Science and Innovation: Vectors of Development: Collection of Scientific Articles in 2 vol., International Scientific and Practical Conference of Young Scientists, 1, 229-232 (2018)

11. A.V. Zharov, I.V. Ivanov, A.P. Strelnikov, Autopsy and pathomorphological diagnostics of animal diseases, 400 (2000)

12. A.F. Klimov, A. I. Akayevsky, Pet Anatomy, 1040 (2011)

13. G.G. Avtandilov, Medical morphometry: manual, 384 (1990)

14. M. S. Kirnos, L.V. Tkachenko, Youth - to Barnaul, Materials of XX city scientificpractical conference of young scientists, 554 (2019) 